\title{
Safety Analysis of Adjuvant Chemotherapy with Docetaxel Administered with or without Anthracyclines to Early Stage Breast Cancer Patients: Combined Results from the Asia- Pacific Breast Initiatives I and II
}

\author{
Sung Bae Kim ${ }^{1 *}$, Ahmed Sayeed ${ }^{2}$, Antonio H Villalon ${ }^{3}$, Zhen Zhou Shen ${ }^{4}$, Tsz \\ Kok Yau ${ }^{5}$, Mazhar Ali Shah ${ }^{6}$, Meng Feng $\mathrm{Hou}^{7}$, Tran Van Thuan ${ }^{8}$, Duc Nguyen \\ Ba $^{9}$, Tsu-Yi Chao ${ }^{10}$
}

\begin{abstract}
Background: The Asia-Pacific Breast Initiatives (APBI) I and II registries were established to collect safety data for patients with early stage breast cancer receiving adjuvant docetaxel-based regimens in the Asia-Pacific region. Materials and Methods: Data from the two registries were combined to perform a safety analysis. Participants in the registry were women with early stage operable breast cancer with an intermediate or high risk of recurrence. These women received adjuvant chemotherapy that included docetaxel between 2006 and 2011. Adverse events (AEs) were recorded and analyzed. Results: Data were collected from 3,224 patients from 13 countries. The mean dose intensity of docetaxel was $24.1,22.7,25.1 \mathrm{mg} / \mathrm{m}^{2} /$ week among patients receiving docetaxel-based monotherapy, combination therapy and sequential therapy, respectively. Granulocyte colony-stimulating factor (G-CSF) was given with docetaxel to $41.8 \%$ of women and $20.6 \%$ of women receiving prophylactic antibiotics. Adverse events were reported in $86 \%$ of patients (anthracycline-containing regimens vs. non-anthracycline regimens; $87 \%$ vs. $80 \%$ ). The most common adverse events were alopecia, nausea, neutropenia, vomiting, and myalgia. Adverse events NCI CTCAE $\geq$ Grade 3 were reported in $45.4 \%$ of patients. Serious adverse events were reported in $13 \%$ of patients, of which $2.5 \%$ led to study discontinuation. Forty-six deaths $(1.4 \%)$ were reported, with no significant difference between regimens. Conclusions: The safety parameters of adjuvant docetaxel therapy used to treat sequential Asian women were comparable to those reported in clinical trials evaluating the role of adjuvant docetaxel. No unusual adverse events linked to Asia-Pacific region patients were observed.
\end{abstract}

Keywords: Adjuvant therapy - docetaxel - early breast cancer - taxanes

Asian Pac J Cancer Prev, 17 (2), 697-702

\section{Introduction}

Breast cancer is the most common cancer among women in Asian countries. Although the incidence of breast cancer in Asia is lower than in Western countries, it is increasing at a rapid rate with diet and lifestyle changing (Yip, 2009). Surgery followed by adjuvant systemic therapy is recommended for most patients with early invasive breast cancer, irrespective of age (National Collaborating Centre for Cancer, 2009).

Adjuvant chemotherapy has been reported to improve survival in women with early stage breast cancer (EBCTCG, 2005). The taxanes docetaxel or paclitaxel, are important components of adjuvant chemotherapy for early breast cancer (EBC), metastatic breast cancer, and preoperative therapy. The efficacy of taxane-containing regimens has been established in large randomized studies. Doxorubicin and docetaxel (AT) or docetaxel, doxorubicin and cyclophosphamide (TAC) were shown to improve relapse-free and overall survival compared to regimens not containing taxanes. A metanalysis comparing any taxane-plus -based regimen with same or more non-taxane chemotherapy $(n=44,000)$ reported addition of taxanes to the anthracycline based regimen results in the reduction of breast cancer (RR 0.86, SE 0.04, p<0.0005) (EBCTCG, 2005; Fumoleau et al., 2005; Pant et al., 2008). Previous studies have reported the occurrence of neutropenic events as one of the major adverse events associated with

${ }^{1}$ Asan Medical Center, University of Ulsan College of Medicine, Seoul, Korea,${ }^{2}$ Holy Family Red Crescent Medical College Hospital, Dhaka, Bangladesh, ${ }^{3}$ Manila Doctors Hospital, Manila, Philippines, ${ }^{4}$ Shanghai Fudan University Cancer Hospital, Shanghai, China, ${ }^{5}$ Pamela Youde Nethersole Eastern Hospital, Hong Kong, ${ }^{6}$ Shaukat Khanum Memorial Cancer Hospital and Research Centre, Lahore, Pakistan, ${ }^{7}$ Kaohsiung Medical University, Taiwan, ${ }^{8} \mathrm{~K}$ Institute (National Cancer Hospital), ${ }^{9}$ National Cancer Hospital, Vietnam, ${ }^{10}$ Tri-Service General Hospital, Taiwan, *For correspondence: sbkim3@amc.seoul.kr 
administration of docetaxel in the adjuvant setting (Martin et al., 2005). Grade 4 neutropenia is observed in $85 \%$ of patients at the dose of $100 \mathrm{mg} / \mathrm{m} 2.5$ and the severity of neutropenia has been found to be dependent on the extent of prior therapy (Pant et al., 2008). Neutropenia related to chemotherapy is associated with considerable morbidity and mortality (Bordoni et al., 2012). Furthermore, the appearance of grade 3 or grade 4 neutropenia can lead to delayed chemotherapy administration or dose reduction, which in turn can cause poor clinical outcomes (Younus et al., 2012).

Since the 1990s, anthracyclines have been considered essential drugs for adjuvant chemotherapy in the management of EBC (Lu et al., 2004). However, anthracycline-based therapies may produce complications such as risk of delayed, life-threatening, dilatory congestive heart failure (CHF) and secondary leukaemia (Tack et al., 2004). Since breast cancer is often diagnosed at an earlier age in women in the Asia-Pacific region, it is important to assess the risk of delayed CHF in that population (de Boer et al., 2011). It has been reported that combined taxane and anthracycline therapy leads to improved outcome11, but the absence of cardiac toxicity from the use of taxanes has led to the assessment of noncontaining chemotherapy in HER2/neu-positive (with trastuzumab) and in HER2/neu-negative breast cancer (de Boer et al., 2011).

Clinical trials are restricted in their patient selection and treatment strategies, whereas in real-world conditions the patient population is heterogeneous and treatment strategy varies from patient to patient and clinician to clinician. Epidemiological studies have revealed key differences in disease characteristics and treatment response between Western and Eastern women with breast cancer (Grenade, et. al., 2014). Until recently no large scale real-world studies have been conducted to evaluate the safety, and efficacy of taxanes as a part of adjuvant therapy for breast cancer in the Asia-Pacific population in a real-world setting.

The Asia-Pacific Breast Initiative (APBI) - I and II registries were set up to collect, analyze and disseminate data for real-world patients from the Asia-Pacific region with early-stage breast cancer treated with docetaxel, either with or without an anthracycline, as adjuvant chemotherapy. The goal was to improve the quality of care of these patients through a better understanding of treatment patterns and outcomes within individual countries and in the region. Here we report the combined safety analysis based on data pooled from the APBI-I and -II registries to evaluate the safety of adjuvant chemotherapy regimens, including docetaxel in the AsiaPacific region.

\section{Materials and Methods}

\section{Study Design}

The APBI-I (2006 - 2008)and APBI-II (2009 - 2011) registries were phase IV, observational, open-label, longitudinal, multi-centre, multi-country studies that prospectively enrolled patients with operable breast cancer with either a high risk or intermediate-to-high risk of recurrence who were administered a range of docetaxel-based treatment regimens (table 1). All patients received commercial docetaxel (Taxotere), and the drug was reconstituted to the manufacturer's guidelines. This study was funded by Sanofi Aventis.

Patients aged $>18$ years with newly diagnosed early stage breast cancer were enrolled consecutively. The treatment was determined by the respective consulting physicians. Exclusion criteria were billirubin $>$ upper limit of normal (ULN), serum glutamic oxaloacetic transaminase (SGOT) and/or serum glutamic pyruvic transaminase $($ SGPT) $>1.5 \times$ ULN concomitant with alkaline phosphatase $>2.5 \times \mathrm{ULN}$, neutrophil counts of $<1500$ cells $/ \mathrm{mm} 3$ and history of severe hypersensitivity reactions to docetaxel or to other drugs formulated with polysorbate 80 . The data acquired in these registries reflect a 'real world' approach to the treatment of patients with early stage breast cancer. The safety data were collected after 1 year of follow up for the APBI-I registry and after 1.5 years follow up for the APBI-II registry.

Consistent with the aims of these observational studies, there were no protocol-specified management directions beyond the intention to treat with docetaxel-based chemotherapy: there were no mandated interventions and patients were treated as per the discretion of the treating physician. Approval for the protocols and all amendments was obtained from independent ethics committees and/or institutional review boards of the respective study centres. Written informed consent for data release was obtained from the patients.

The pooled patient data from both the registries were broadly categorized into two groups -anthracycline group and non-anthracycline group, to compare and evaluate differences between the use of docetaxel with or without anthracycline-based regimens.

Docetaxel-based regimens were divided into five categories: docetaxel monotherapy, docetaxel combination therapy, docetaxel sequential therapy, docetaxeltrastuzumab combination therapy, and docetaxeltrastuzumab sequential therapy. Toxicity and AEs were recorded during each cycle, at the end of chemotherapy and during the follow-up period. Use of concomitant medications such as hormone therapy and radiotherapy were recorded at inclusion during each cycle, at the end of chemotherapy and during follow-up. Disease status (in APBI-I only) and survival status were assessed at the end of chemotherapy and at follow-up. Data from Multi Gated Acquisition Scan (MUGA) or 2D echo and serum cholesterol levels were assessed in APBI-II only (data not shown). All reported deaths were summarized according to chemotherapy category and cause of death.

\section{Overall Chemotherapy Exposure}

For each category of actual chemotherapy, the following parameters were summarized for different countries: total number of cycles administered (excluding trastuzumab only cycles), duration of chemotherapy (days), details of drugs administered and actual dose (mg) per cycle and dose intensity. Dose intensity was calculated based on the dose given per unit body surface area and duration of the exposure. 


\section{Safety parameters and follow up}

Safety outcomes were evaluated during the course of treatment in this registry. In this study, all AEs/ serious adverse events (SAEs), regardless of relationship to the chemotherapy, spanning from the first day of chemotherapy, 30 days after last chemotherapy and at the follow up visit were recorded. Investigators identified the worst grade (according to National Cancer Institute Common Terminology Criteria for Adverse Events [NCICTCAE v3]), seriousness criteria, relationship, action taken with chemotherapy, corrective treatment/therapy, and whether AEs resolved before the next cycle of chemotherapy. Febrile neutropenia was reported as a fever of unknown origin without clinically or microbiologically documented infection as absolute neutrophil count (ANC) $<1.0 \times 10^{9} / \mathrm{L}$, and fever $\geq 38.5^{\circ} \mathrm{C}$. Neutropenia was reported as $\mathrm{ANC}<1.0 \times 10^{9} / \mathrm{L}$

\section{Statistical analysis}

Data have been analyzed and presented as mean \pm standard deviation, median, interquartile range [IQR], percentage, and a Student's t-test. Kaplan-Meier methods were used to estimate cumulative events rates. All statistical analyses have been performed at the $95 \%$ significance level.

\section{Results}

\section{Patient Disposition}

A total of 3400 patients from 13 countries in the AsiaPacific region were included in the APBI-I and APBI-II registries (Figure 1) of whom a majority were from Korea (26.3\%), Taiwan $(24.8 \%)$ and China (18.1\%).

In this pooled data, the safety analysis included 3224 patients ( $94.8 \%$ of the overall population). One hundred and seventy six patients were excluded from the analysis due to various reasons such as not receiving docetaxel during chemotherapy (55.1\%), informed consent obtained after first docetaxel administration (21\%), not receiving any chemotherapy $(13.1 \%)$, not meeting eligibility criteria $(13.1 \%)$, and $2.3 \%$ did not receive branded taxotere.

\section{Chemotherapy Regimens}

Anthracycline-containing regimens (doxorubicin, epirubicin or pirarubicin) were administered to $85.3 \%$ $(n=2751)$ of patients while non-anthracycline-containing regimens were administered to $14.7 \%(n=473)$ of patients.

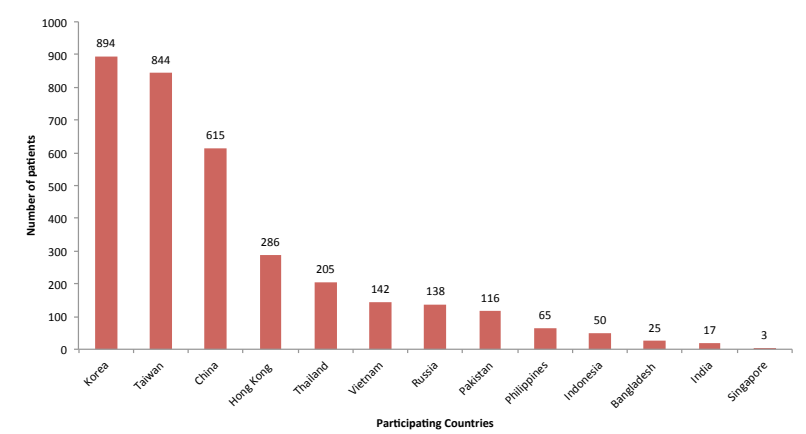

Figure 1. Number of Patients Enrolled in the APBI-I and - APBI-II registries $(n=3224)$
The different chemotherapy regimens employed with mean dose intensity of docetaxel are shown in table 2 . The mean dose intensity for docetaxel was between 22.7-25.1 $\mathrm{mg} / \mathrm{m} 2 /$ week for different regimen group.

The mean number of cycles with docetaxel ranged from approximately 4 to 8 in the overall population, and in the anthracycline as well as non-anthracycline groups as described in figure 2 . The average docetaxel cycles administered were, 5.3 in docetaxel monotherapy, 5.4 in docetaxel combination therapy, 3.8 in docetaxel sequential therapy, 6 in docetaxel-Herceptin combination therapy, and 4.1 in docetaxel, Herceptin sequential therapy. Dose modifications (both increases and decreases) were required, frequently. Most patients did receive the intended total actual dose of docetaxel, whereas less than half of them were maintained at the intended dose intensity.

\section{Other Therapies}

About $53 \%$ of patients received radiation therapy following adjuvant chemotherapy while hormonal therapy was given to $60 \%$ patients (usually tamoxifen) during follow-up. Some concomitant chemotherapy supportive medication was administered to most of the patients (98$100 \%$ patients).

\section{Safety Adverse Events}

Adverse Events were reported in $85.5 \%$ of patients. The frequency of several of AEs including deaths, was broadly comparable across all chemotherapy strategies. Overall, a higher number of AEs related to chemotherapy were noted with anthracycline regimens (86.6\%) compared to non-anthracycline regimens $(79.7 \%)$. The AEs in docetaxel combination and sequential therapy were comparable at $82.2 \%$ and $88.3 \%$, respectively. The incidence of AEs related to chemotherapy in both regimens is depicted in Figure 3.

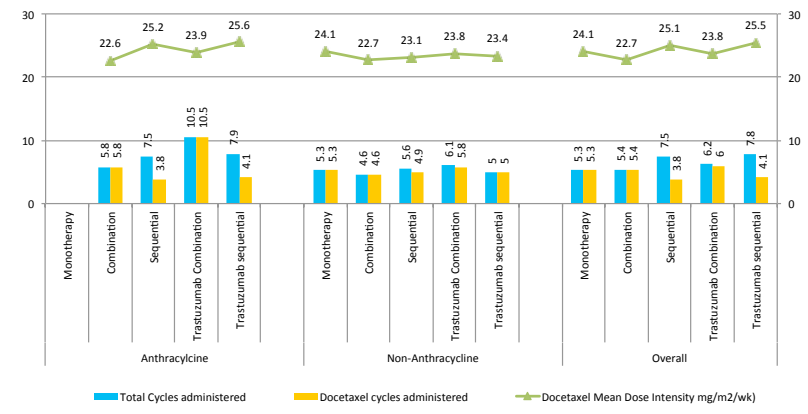

Figure 2. Docetaxel Exposure (safety population)

Patient experienced adverse events across different therapies

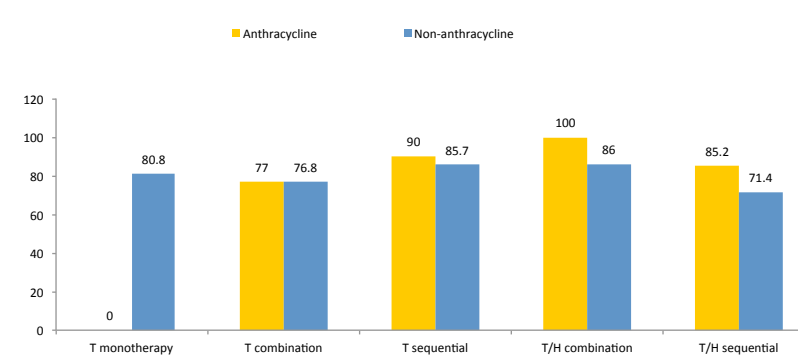

Figure 3. Number of Patients (\%) Who Experienced Adverse Events across Different Docetaxel Based Regimen 
Kim Sung Bae et al

Non-anthracycline containing regimens, compared to the anthracycline regimens, were associated with a higher incidence of decreased leukocytes and a lower frequency of alopecia, nausea, decreased neutrophil, vomiting, myalgia, stomatitis, anemia, constipation, neurosensory effects, anorexia, asthenia, nail changes, insomnia, oral cavity mucositis, peripheral edema, and febrile neutropenia (Figure 4).

NCI CTCAE $\geq$ Grade 3 events: Anthracycline vs. nonanthracycline

National Cancer Institute-Common Terminology Criteria for Adverse Events $\geq$ Grade 3 events were noted in $45.4 \%$ of patients and were higher in the anthracycline group (46.7\%) compared to the non-anthracycline group $(37.4 \%)$. AEs that were related to chemotherapy were observed in $44.2 \%$ of patients (anthracycline group vs. non-anthracycline; $45.4 \%$ vs. $37.6 \%$ ). In contrast the anthracycline regimens were associated with higher frequency of neutropenia (32.6\% vs. $22.6 \%)$, febrile neutropenia ( $10.6 \%$ vs. $6.8 \%)$, vomiting ( $3.6 \%$ vs. $0.8 \%)$ and stomatitis $(0.8 \%$ vs. $0.2 \%)$ in comparison to nonanthracycline regimens.

The mean number of cycles in which grades 3-4 neutropenia was reported, was 3.0 (95\% confidence interval $[\mathrm{CI}], 2.9-3.1): 3.1$ (95\% CI, 3.0-3.2) for anthracycline containing regimens vs. 2.5 (95\% CI, 2.22.8 ) for non-anthracycline-containing regimens. The mean (95\% CI) number of cycles in which febrile neutropenia occurred was 1.6 cycles (95\% CI, 1.5, - 1.7) across both regimen groups.

Of the chemotherapy support medications administered, $35.6 \%$ and $19.7 \%$ of the overall patient population received granulocyte colony-stimulating factor (G-CSF) and antibiotics, respectively. Of the patients receiving anthracycline-containing regimen, $41.8 \%$ patients were administered G-CSF and $21.6 \%$ of patients received antibiotics. Of the patients receiving non-anthracyclinecontaining regimen, $41.2 \%$ patients received G-CSF and $14.1 \%$ received antibiotics.

Patients were assumed to have febrile neutropenia if they had taken any medication that is linked to febrile neutropenia. Patients were treated for adverse events defined by the National Cancer Institute-Common Terminology Criteria for Adverse Events. Patients were counted for prophylaxis with febrile neutropenia if an indication of febrile neutropenia was captured for the medication or if the patient experienced an adverse event of febrile neutropenia in the same cycle in which the drug was administered As depicted in fig. 5, more patients received growth factors prophylactically than concomitantly for febrile neutropenia.

\section{Combination vs. sequential docetaxel therapy}

The group receiving sequential therapy with docetaxel had a higher incidence $(46.5 \%)$ of NCI CTCAE $\geq$ Grade 3 AEs compared to the monotherapy and combination arms (34.6\% and 44\%). Exploratory analysis revealed that the number of patients receiving G-CSF was higher in the combination arm $(65.2 \%)$ compared to the arm receiving sequential therapy (25.9\%) with docetaxel. Antibiotics

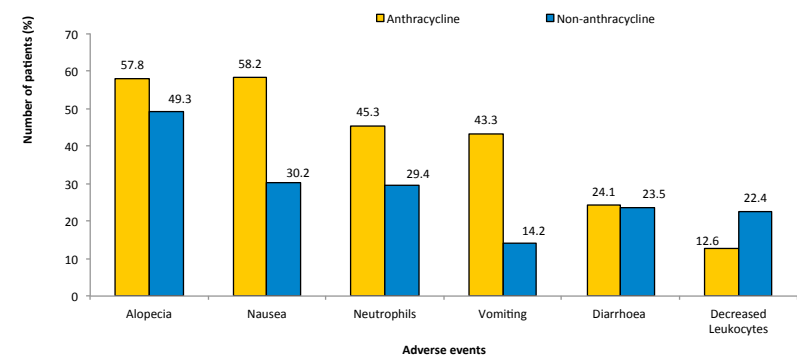

Figure 4. Five most frequently noted AEs across docetaxel-based anthracycline and non-anthracycline regimens

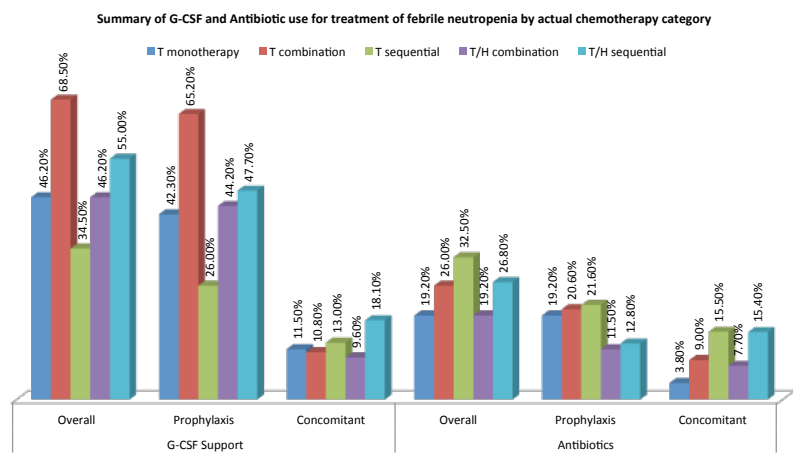

Figure 5. Administration of Growth Factors Including G-CSF and Antibiotics for the Treatment Febrile Neutropenia

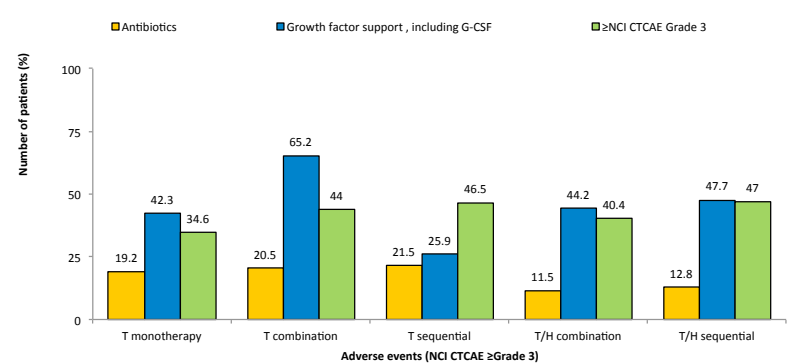

Figure 6. Number of Patients (\%) who Experienced NCI CTCAE $\geq$ Grade 3 Events Across Different Docetaxel Therapies and use of Supportive Medications

were administered to $20.5 \%$ and $21.5 \%$ of patients in these categories, respectively (figure 6).

\section{Deaths}

Overall, 46 deaths (1.4\%) were reported. Death was reported in $1.8 \%$ of patients in the docetaxel combination regimen, $1.3 \%$ of docetaxel sequential patients, $1.9 \%$ of docetaxel/trastuzuamab combination patients and $0.7 \%$ of docetaxel/trastuzumab sequential patients.

Two patients $(4.3 \%)$ died, this can be related to chemotherapy regimen (anthracycline vs. non anthracycline; $4.9 \%$ vs. 0 ). Deaths due to chemotherapyrelated toxicity accounted for $9.5 \%$ of deaths among the docetaxel combination patients (this was not seen in other chemotherapy regimen groups).

\section{Serious Adverse Events}

Overall, SAEs were reported in about $13 \%$ of patients (13\% in anthracycline vs. $12.7 \%$ in non-anthracycline regimens). About $12 \%$ of patients (12.3\% vs. $11.4 \%$ ) 
reported SAEs that were related to chemotherapy. Although, the incidence of SAE's was higher in the nonanthracycline regimen, no significant difference was found between the anthracycline and non-anthracycline regimens (Table 3). SAEs reported in $11.3 \%$ patients receiving docetaxel combination therapy as compared to $14.2 \%$ of patients receiving docetaxel sequential therapy.

\section{Treatment Discontinuation}

Overall, 85 (2.6\%) patients, who had at least one AE, discontinued therapy. A higher rate of AEs leading to chemotherapy discontinuation was noted with docetaxel monotherapy compared to all other regimens $(7.7 \%$ vs. $\leq 3.0 \%$, respectively). The percentage of patients who discontinued therapy after at least one AE was $2.8 \%$ for those receiving a docetaxel combination regimen compared to $2 \%$ for those receiving a docetaxel sequential regimen. Discontinuation due to AEs related to chemotherapy was reported in $2.5 \%$ of patients in both groups of anthracycline- and non-anthracycline-containing regimens. Some of the patients who discontinued treatment received prophylactic G-CSF $(0.78 \%, \mathrm{~N}=25)$ or antibiotics $(0.74 \%, \mathrm{~N}=24)$.

\section{Discussion}

Historically, the Asia-Pacific region has witnessed a far lower incidence of breast cancer compared to developed Western countries. However, in the last two decades breast cancer is increasing in the region (Grenade, et. al., 2014). Eastern and Western patient populations have shown significant differences in breast cancer epidemiology (Grenade, et. al., 2014). Owing to differences in the epidemiology of disease, it is necessary to study the safety and efficacy of routine treatment strategies in Eastern populations. However, no prospective study has been conducted to study the efficacy and safety of commonly practiced chemotherapy regimens in Eastern patient populations. To fill this gap, APBI-I and APBI-II were established.

Docetaxel has emerged as a frequently-used chemotherapeutic agent for EBC patients (Goble, et. al., 2014). Various docetaxel-based treatment strategies were evaluated for their safety in anthracycline and non-anthracycline-based regimens in these registries. In this manuscript, safety data are reported from a pooled analysis from both the registries for 3,224 patients from 13 countries of the region.

The mean dose intensity of docetaxel was 22.7 and $25.5 \mathrm{mg} / \mathrm{m}^{2} /$ week in anthracycline and nonanthracycline containing regimens spanning 4-8 cycles. Sequential therapy was more commonly used than combination regimens. The most frequently used anthracycline regimens included TEC (docetaxel, epirubicin, cyclophosphamide) and AC-T (adriamycin, cyclophosphamide, and docetaxel). The most frequently used non-anthracycline regimens were TC (docetaxel and cyclophosphamide) and $\mathrm{TCbH}$ (docetaxel. carboplatin and trastuzumab). No study or registry to date has ever reported the real life usage of regimens related to docetaxel usage in detail.
AEs, NCI CTCAE $\geq$ Grade 3 AEs, and SAEs observed in APBI registries were in accord with previously published reports evaluating the safety profile of docetaxel in various chemotherapeutic regimens. The AEs were reported in $85.5 \%$ of patients and were numerically higher among patients receiving anthracycline-containing regimens compared to non-anthracycline regimens $(86.6 \%$ vs. $79.7 \%$, respectively) in this study. However, nonfatal adverse events were under-reported compared to other trials. The nature and the incidence of each of the AEs varied based on the (nature of the) regimen used. The higher incidence of AEs and dropout rate in the monotherapy docetaxel arm can be attributed to the fact that monotherapy was prescribed for very sick patients who would not have been able to tolerate combination therapies.

Neutropenia and febrile neutropenia were the most common NCI CTCAE $\geq$ Grade 3 AEs observed in this registry. Both the adverse events have been reported in phase III BCIRG 001 and GeparTrio Trial (Martin, et. al., 2005; von, et. al., 2008) although in both studies only TAC the regimen was used whereas APBI studies included several regimen combinations containing docetaxel.

Neutropenia and febrile neutropenia were the most common NCI CTCAE $\geq$ Grade 3 AEs observed in this registry. Both the adverse events have been reported in phase III BCIRG 001 and GeparTrio Trial (Martin, et. al., 2005; von, et. al., 2008) although in both studies only TAC the regimen was used whereas APBI studies included several regimen combinations containing docetaxel. Neutropenia ( $\geq$ Grade 3 ) was reported in $32.6 \%$ of patients receiving anthracycline regimen in these registries compared to $65.5 \%$ in the BCIRG 001 study and $42.1 \%$ in the GeparTrio trial. The incidence of $\geq$ Grade 3 febrile neutropenia was observed in $10.6 \%$ of patients receiving anthracycline regimens as compared to $24.7 \%$ reported in BCIRG001 study and 7.4\% in GeparTrio trial. A few gastroenterological $\geq$ Grade $3 \mathrm{AE}$ were also reported in $<5 \%$ of patients. APBI registries reported lower incidence of $\geq$ Grade 3 neutropenia as compared to BCIRG001 and GeparTrio trial. However, febrile neutropenia was reported lower than BCIRG001 trial. But, it was slightly higher than GeparTrio trial. Taken together, observations from APBI registries showed docetaxel as a safe adjuvant in anthracycline- and non-anthracycline-based chemotherapy for EBC patients.

SAEs were reported in approximately $13 \%$ of patients. The most frequently reported SAEs $(\geq 0.5 \%$ of patients) were febrile neutropenia, neutropenia, and leukopenia. Non-anthracycline-containing regimens were associated with a higher frequency of SAEs of leukopenia, diarrhoea, and myalgia, and with a lower frequency of febrile neutropenia and neutropenia. Investigators reported only $4.3 \%$ of deaths due to chemotherapy (anthracycline vs. non-anthracycline; $4.9 \%$ vs. 0 ). Most deaths were recorded in the docetaxel sequential arm than in any other regimen group. A phase III clinical trial by Jones et al (2009) reported less than $1 \%$ deaths due to chemotherapy on the administration of TC regimen in a 66-month, 1016-patients study. In the BCIRG001 trial, $0.3 \%$ deaths were reported due to chemotherapy at 55 months follow up. However, the 
higher death rate in APBI registries cannot be attributed to one factor due to great diversity of patient population and treatment. Furthermore, the discontinuation rates due to chemotherapy-related side-effects were low, with $2.6 \%$ of the patients discontinuing the therapy.

The number of patients who completed all the treatment cycles but required G-CSF support was higher in combination therapy $(23.5 \%)$ compared to $18.3 \%$ in docetaxel sequential therapy. Integration of G-CSF prophylactically or concomitantly has been reported to reduce neutropenia (Bordoni , et. al., 2012; Younus, et. al., 2012). In the GEICAM 9805 study, primary G-CSF support compared to secondary G-CSF prophylaxis, was not found to cause an improvement in median dose intensity or cumulative dose, but was associated with significantly more patients completing six cycles of therapy (Martín, et. al., 2010). In these registries, the use of G-CSF was the highest in the group receiving combination therapy with docetaxel. In contrast, the group receiving sequential therapy with docetaxel had the lowest use of G-CSF, but had a higher incidence of Grade 3 AEs compared to the other groups. Nonetheless, specific safety data collection pertaining to G-CSF administration was beyond the scope of these registries.

\section{Limitations}

Granulocyte colony-stimulating factor has been reported to ameliorate the safety profile (neutropenia and febrile neutropenia) of various chemotherapeutic regimens including docetaxel. Since the focus of this combined analysis of the two registries APBI-I and APBI-II was to collect data regarding docetaxel in anthracycline- and non-anthracycline-based therapy in the Asia-Pacific population, it does not report the effects of supportive medications such as antibiotics and G-CSF prophylactically for treatment. Neutropenia is measured in blood counts mid-cycle. However, this is a real-life drug registry and the time points for blood count could not be made mandatory. Hence, data regarding neutropenia may not represent the actual incidence of neutropenia in study population. Moreover, cardiac safety associated with the use of anthracyclines parameters such as LVEF was not reported in APBI-I.

In conclusion, The analysis from APBI-I and APBI-II registries indicate the safety profile of adjuvant docetaxel therapy to be comparable across anthracycline- and nonanthracycline-containing chemotherapy regimens in the Asia-Pacific population in real-world settings. No new significant AEs and/or SAEs were observed in the patients of the Asia-Pacific region using docetaxel-based chemotherapy. Moreover, safety parameters were also comparable between anthracycline- and non-containing chemotherapy regimens either in combination or sequential regimen. Hence, the findings from this registry indicate that in current clinical settings, docetaxel-based therapies may be employed safely across the Asia-Pacific region on the basis of the experience of treating physicians. The safety profiles observed with various regimens observed in these registries can help doctors and patients decide to which regimen they would be most comfortable with. Furthermore, the impact of the inclusion of G-CSF and antibiotics as supportive medication on the incidence of adverse events needs additional investigation.

\section{Acknowledgements}

We would like to acknowledge Sanofi Aventis for funding this project. Sincere thanks to all the participating investigators, clinical trial staff and others who supported the conduct of this study.

\section{References}

Bordoni RE, Haislip ST, Gilmore JW, et al (2012). Estimation of the incidence of febrile neutropenia in women receiving docetaxel plus cyclophosphamide as adjuvant therapy for early-stage breast cancer: a large community-based retrospective study. Commun Oncol, 9, 158-64.

de Boer RH, Chan A, Tran B, et al (2011). Use of nonanthracycline regimens in early stage breast cancer in Australia. Asia Pac J Clin Oncol, 7, 4-10.

Early Breast Cancer Trialists' Collaborative Group (EBCTCG) (2005). Effects of chemotherapy and hormonal therapy for early breast cancer on recurrence and 15-year survival: an overview of the randomised trials. Lancet, 365, 1687-717.

Fumoleau P, Roche H (2005). The Impact of docetaxel on Adjuvant Breast Cancer. Business Briefing: Eur Oncol Rev, 1,1 .

Goble S, Bear HD (2003). Emerging role of taxanes in adjuvant and neoadjuvant therapy for breast cancer: the potential and the questions. Surg Clin North Am, 83, 943-971

Grenade C, Phelps MA, Villalona-Calero MA (2014). Race and ethnicity in cancer therapy: what have we learned? Clin Pharmacol Ther, 95, 403-12

Jones S, Holmes FA, O'Shaughnessy J, et al (2009). Docetaxel With Cyclophosphamide Is Associated With an Overall Survival Benefit Compared With Doxorubicin and Cyclophosphamide: 7-Year Follow-Up of US Oncology Research Trial 9735. J Clin Oncol, 27, 1177-83.

Lu YS, Kuo SH, Huang CS (2004). Recent advances in the management of primary breast cancers. J Formos Med Assoc, 103, 579-98.

Martin M, Pienkowski T, Mackey J, et al (2005). Adjuvant docetaxel for node-positive breast cancer. $N$ Engl J Med, 352, 2302-13

Martín M, Seguí MA, Antón A, et al (2010). Adjuvant docetaxel for high-risk, node-negative breast cancer. $N$ Engl J Med, 363, 2200-10.

National Collaborating Centre for Cancer (2009). NICE clinical guideline 80: Early and locally advanced breast cancer Diagnosis and treatment.

Pant S, Chilukuri M, Ramaswamy B (2008). Docetaxel for the post-surgery treatment of patients with node-positive breast cancer. Ther Clin Risk Manag, 4, 419-24.

Tack DK, Palmieri FM, Perez EA (2004). Anthracycline vs nonanthracycline adjuvant therapy for breast cancer. Oncol (Williston Park), 18, 1378-81.

von Minckwitz G, Kümmel S, Vogel P, et al (2008). German Breast Group, Intensified neoadjuvant chemotherapy in earlyresponding breast cancer: phase III randomized GeparTrio study. J Natl Cancer Inst, 100, 552-62.

Yip CH (2009). Breast cancer in Asia. Methods Mol Biol, 471, 51-64.

Younus J, Vandenberg T, Jawaid M (2012). Febrile neutropenia rates with adjuvant docetaxel and cyclophosphamide chemotherapy in early breast cancer: discrepancy between published reports and community practice-an updated analysis. Curr OncoL, 19, 332-4. 